\title{
Conventions used in transcription and glossing
}

\author{
- $\quad$ morpheme break \\ $>\quad$ acting on \\ $=\quad$ clitic boundary \\ ... follow-on utterance \\ separates categories encoded by \\ a portmanteau morpheme
}

\section{Conventions used to indicate source of data}

Most of the Gurindji examples are accompanied by a reference containing certain information shown schematically below:

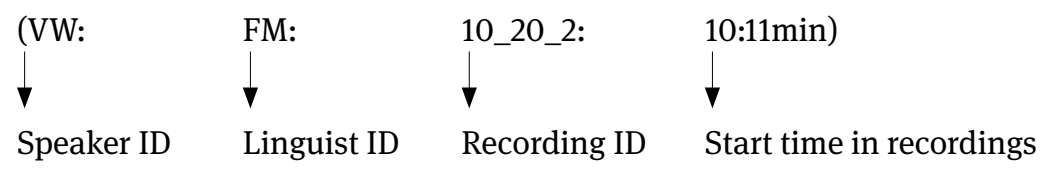

Often the recording ID contains information about the year the recording was made e.g. FM10_20_2 was recorded in 2010. Note that many of the sentences from Patrick McConvell's work do not have an archive reference. These sentences came from the original sketch grammar and are unable to be matched with an original recording.

Speaker ID:

$\begin{array}{ll}\text { BB } & \text { Blanche Bulngari } \\ \text { BR } & \text { Banjo Ryan } \\ \text { BW } & \text { Biddy Wavehill } \\ \text { CE } & \text { Cecelia Edwards } \\ \text { CN } & \text { Connie Ngarrmaya } \\ \text { DB } & \text { Doris Butler } \\ \text { DD } & \text { Dandy Danbayarri } \\ \text { DW } & \text { Doris Warnmal } \\ \text { EO } & \text { Ena Oscar } \\ \text { IM } & \text { Ida Malyik } \\ \text { JK } & \text { Johnny Kijngayarri } \\ \text { KM } & \text { Kitty Mintawurr } \\ \text { PN } & \text { Pincher Nyurrmiari } \\ \text { PP } & \text { Peanut Pontiari } \\ \text { RW } & \text { Ronnie Wavehill } \\ \text { SM } & \text { Smiler Major } \\ \text { SO } & \text { Sarah Oscar }\end{array}$


$\mathbf{X X X}$

Conventions used in transcription and glossing

TD Topsy Dodd Ngarnjal

TN Tommy Ngaliwin

VW Violet Wadrill

Linguist ID (see §1.2.1):

EC Erika Charola

FM Felicity Meakins

$\mathrm{KH} \quad$ Ken Hale

LC Lauren Campbell

MCCONVELL Patrick McConvell

MCNAIR Helen and Norm McNair 